\title{
Cyclic Vomiting Syndrome (CVS): is there a difference based on onset of symptoms - pediatric versus adult?
}

Nilay Kumar ${ }^{1}$, Qumseya Bashar ${ }^{2}$, Naveen Reddy ${ }^{3}$, Jyotirmoy Sengupta', Ashwin Ananthakrishnan ${ }^{4}$, Abigail Schroeder ${ }^{1}$, Walter J Hogan ${ }^{1}$ and Thangam Venkatesan ${ }^{1^{*}}$

\begin{abstract}
Background: Cyclic Vomiting Syndrome (CVS) is a well-recognized functional gastrointestinal disorder in children but its presentation is poorly understood in adults. Genetic differences in pediatric-onset (presentation before age 18) and adult-onset CVS have been reported recently but their clinical features and possible differences in response to therapy have not been well studied.
\end{abstract}

Methods: This was a retrospective review of 101 CVS patients seen at the Medical College of Wisconsin between 2006 and 2008. Rome III criteria were utilized to make the diagnosis of CVS.

Results: Our study population comprised of 29(29\%) pediatric-onset and 72 (71\%) adult-onset CVS patients. Pediatric-onset CVS patients were more likely to be female ( $86 \%$ vs. $57 \%, p=0.005)$ and had a higher prevalence of CVS plus (CVS + neurocognitive disorders) as compared to adult-onset CVS patients ( $14 \%$ vs. $3 \%, p=0.05)$. There was a longer delay in diagnosis ( $10 \pm 7$ years) in the pediatric-onset group when compared to (5 \pm 7 years) adultonset CVS group $(p=0.001)$. Chronic opiate use was less frequent in the pediatric-onset group compared to adultonset patients ( $0 \%$ vs. $23 \%, p=0.004)$. Aside from these differences, the two groups were similar with regards to their clinical features and the time of onset of symptoms did not predict response to standard treatment. The majority of patients (86\%) responded to treatment with tricyclic antidepressants, anticonvulsants (topiramate), coenzyme Q-10, and L-carnitine. Non-response to therapy was associated with coalescence of symptoms, chronic opiate use and more severe disease as characterized by longer episodes, greater number of emergency department visits in the year prior to presentation, presence of disability and non-compliance on univariate analysis. On multivariate analysis, only compliance to therapy was associated with a response. (88\% vs. 38\%, Odds Ratio, OR 9.6; 95\% Confidence Interval [Cl], 1.18-77.05).

Conclusion: Despite reported genetic differences, the clinical features and response to standard therapy in pediatric- and adult-onset CVS were mostly similar. Most patients (86\%) responded to therapy and compliance was the only factor associated with a response.

Keywords: Motility, Cyclic vomiting, Nausea, Abdominal pain

\footnotetext{
* Correspondence: tvenkate@mcw.edu

'Department of Gastroenterology and Hepatology, Medical College of Wisconsin, 4th Floor, Froedtert East Building, 9200 W Wisconsin Avenue, Milwaukee, WI 53226, USA

Full list of author information is available at the end of the article
} 


\section{Background}

Cyclic vomiting syndrome (CVS) is a chronic gastrointestinal disorder that was initially described in children but is now being recognized with increasing frequency in adults [1-3]. Rome III criteria for CVS in adults includes stereotypical episodes of vomiting regarding onset (acute) and duration (less than 1 week); 3 or more discrete episodes in the prior year and absence of nausea and vomiting between the episodes [4]. Adult CVS can range from mild disease with infrequent episodes to severe life-disabling disease requiring multiple emergency department (ED) visits and frequent hospitalizations $[1,5,6]$. Since CVS is often unrecognized, these patients often undergo a battery of unnecessary diagnostic and therapeutic procedures without any clinical benefit $[1,7]$. Many patients, if not treated appropriately, develop coalescence of symptoms with interepisodic nausea and loss of the periodicity of episodes. Several CVS patients also undergo unnecessary surgical procedures such as cholecystectomy for symptoms caused by CVS [7].

CVS may be categorized as pediatric- and adult-onset CVS based on the age of onset of symptoms. Pediatriconset CVS patients develop symptoms prior to the age of 18 while adult-onset CVS patients develop symptoms $\geq 18$ years $[1,8]$. Genetic differences have been identified in these two groups with mitochondrial DNA (mtDNA) mutations, $16519 \mathrm{~T}$ and 3010A being associated with pediatric-onset CVS [2,9-11].

Strong maternal inheritance of multiple disease manifestations and abnormal urine organic acids has been demonstrated in children with CVS, suggesting the presence of predisposing mtDNA sequence polymorphisms $[12,13]$. It has also been reported that pediatric-onset CVS patients have a higher proportion of maternal inheritance of functional symptomatology as ascertained by quantitative pedigree analysis [14].There are presently no data that have elucidate the clinical differences and response to treatment pertaining to these groups . Based on our experience with a large cohort of CVS patients seen at the adult CVS clinic in Milwaukee, we hypothesized that despite these genetic differences, both groups of patients had similar clinical characteristics and response to standard medications used in the treatment of CVS. For treatment of these patients we also utilized the standard medical regimen shown to be effective in existing literature [15].

\footnotetext{
Aims

The primary aims of our study were 1) To define the demographics and clinical characteristics and assess response to therapy in pediatric-onset and adult-onset CVS patients. 2) To identify predictors of response to treatment in both groups of patients.
}

\section{Results}

\section{Study population}

Of 101 patients, there were 66 (65\%) females. There were $79(79 \%)$ whites, $17(17 \%)$ blacks, $3(3 \%)$ of hispanic origin and 1 (1\%) of other origin. Six patients met the diagnostic criteria for CVS plus whereas 10 patients met criteria for catamenial CVS (episodes triggered by menstrual periods) [1]. CVS plus is defined as CVS in association with neurocognitive disorders [16]. The mean age at presentation was $27 \pm 12.3$ years. The median duration of an episode was 3 days (range 1-18 days) with 81 (81\%) patients reporting a prodromal phase. At the initial clinic visit, 39 (39\%) patients had coalescence of symptoms with daily or near-daily symptoms but had typical episodes at the outset. Patients with coalescent CVS had a longer delay in diagnosis as compared to typical CVS patients (91 \pm 86 vs. $75 \pm 85$ months) though this was not statistically significant. Of all patients, 63 (62\%) had abdominal pain as a prominent symptom during episodes along with nausea and vomiting. The location of the abdominal pain was variable with 40 patients (63\%) having generalized abdominal pain, 14 patients (22\%) reporting upper abdominal pain and 9 patients (15\%) with pain in the lower abdomen. Almost all patients (90\%) had nausea as a part of their symptom complex and 76 patients (76\%) stated that the onset of episodes was in the morning. Data on hot and cold showers was available in 73 patients; 38 (52\%) patients had symptom relief with hot showers and 1 patient had relief with a cold shower. Hot showers were reported in 25/35 (71\%) CVS patients using marijuana when compared to non users 19/56 (34\%, p = 0.01).

Triggers for CVS episodes were reported in $87(87 \%)$ of patients with the most common triggers being negative stress (unpleasant or sad events) in 67(67\%) and positive stress (happy or exciting events) in 59(59\%). The recovery phase was very variable and ranged from 10 minutes - 7 days. Many patients had concomitant co-morbid conditions such as anxiety (47\%), depression (49\%) and dysautonomia (64\%). Gastric emptying studies were available for review in 40 patients; 10 patients (25\%) had gastroparesis whereas 4 patients (10\%) had rapid gastric emptying and 26 (65\%) had normal emptying.

Thirty of seventy (43\%) patients had a personal history of migraine and $41 / 64(64 \%)$ had a family history of migraine. Most of the patients in our cohort had undergone numerous diagnostic and therapeutic procedures; 28/92 (30\%) had undergone a cholecystectomy for symptoms of CVS without any therapeutic benefit. Eighteen of 75 patients (24\%) reported some form of disability from CVS. Sixteen (24\%) of 66 patients had a delay in higher education and 20 of 70 patients (29\%) reported job loss related to CVS episodes. 
Table 1 Demographic and disease characteristics of patients with CVS based on disease onset

\begin{tabular}{llll}
\hline Variables & $\begin{array}{l}\text { Pediatric Onset } \\
\text { (n-29), }\end{array}$ & $\begin{array}{l}\text { Adult Onset } \\
\text { (n-72) }\end{array}$ & p-value \\
\hline $\begin{array}{l}\text { Mean age at onset } \\
\text { of symptoms(years) }\end{array}$ & $13.4 \pm 12.5$ & $32.3 \pm 12.3$ & $\mathbf{0 . 0 0 0 1}$ \\
$\begin{array}{l}\text { Female sex } \\
\text { Race (\%) }\end{array}$ & $25(86 \%)$ & $41(57 \%)$ & $\mathbf{0 . 0 0 5}$ \\
White & $25(86 \%)$ & $54(75 \%)$ & 0.29 \\
Black & $3(10 \%)$ & $14(20 \%)$ & 0.38 \\
Hispanic & $1(4 \%)$ & $2(3 \%)$ & 1 \\
Other & 0 & $1(1 \%)$ & \\
Type of CVS & & & \\
Cvs plus (\%) & $4(14 \%)$ & $2(3 \%)$ & $\mathbf{0 . 0 5}$ \\
Catamenial CVS & $5(17 \%)$ & $5(7 \%)$ & 0.15 \\
Sato's CVS & $2(7 \%)$ & $4(6 \%)$ & 1 \\
$\begin{array}{l}\text { Delay in diagnosis } \\
\text { (months) }\end{array}$ & $124.75 \pm 85.86$ & $63.53 \pm 85.23$ & $\mathbf{0 . 0 0 1}$ \\
Coalescence & $14(48 \%)$ & $25(36 \%)$ & 0.25
\end{tabular}

Associated Symptoms Available $\mathbf{n}=\mathbf{2 7}$ Available $\mathbf{n}=\mathbf{6 7}$

\begin{tabular}{|c|c|c|c|}
\hline Abdominal Pain & $16(59 \%)$ & $47(70 \%)$ & 0.34 \\
\hline Co-morbid disorders & Available $\mathbf{n}=\mathbf{2 8}$ & Available $n=68$ & \\
\hline IBS & $9(32 \%)$ & $18(27 \%)$ & 0.62 \\
\hline Anxiety & $13(46 \%)$ & $32(47 \%)$ & 0.19 \\
\hline Depression & $9(32 \%)$ & $33(49 \%)$ & 0.18 \\
\hline Bipolar Disorder & $1(4 \%)$ & $1(2 \%)$ & 0.5 \\
\hline Migraine & $16(55 \%)$ & $30(43 \%)$ & 0.28 \\
\hline Cholecystectomy & $9(32 \%)$ & $19(30 \%)$ & 0.81 \\
\hline Family History (\%) & Available $\mathrm{n}=\mathbf{2 5}$ & Available $n=64$ & \\
\hline $\begin{array}{l}\text { Family history of } \\
\text { migraine }\end{array}$ & $16(64 \%)$ & $41(64 \%)$ & 1 \\
\hline Drug use \% & Available $\mathbf{n}=\mathbf{2 7}$ & Available $n=65$ & \\
\hline Marijuana & $8(30 \%)$ & $27(42 \%)$ & 0.35 \\
\hline Alcohol & $7(26 \%)$ & $10(15 \%)$ & 0.25 \\
\hline Tobacco & $3(12 \%)$ & $30(46 \%)$ & 0.001 \\
\hline Narcotics & 0 & $15(23 \%)$ & 0.004 \\
\hline Response to therapy & 19/20 (95\%) & $47 / 56(84 \%)$ & 0.27 \\
\hline
\end{tabular}

\section{Pediatric- vs. adult-onset CVS}

In our cohort of 101 adults with CVS, twenty nine patients (29\%) had pediatric-onset CVS as compared to 72 (71 \%) with adult-onset CVS. The demographics, clinical characteristics, associated co-morbid conditions and response to treatment are depicted in table 1. Patients were predominantly white in both groups but more patients were female in those with pediatric-onset CVS 25 (86 \%) than adult-onset CVS 41(57 \%), $(\mathrm{p}=0.005)$. Both subgroups had similar disease characteristics with no significant differences noted in trigger factors, co-morbid conditions, rate of surgery for CVS symptoms, or personal/family history of migraine. Coalescence of symptoms was noted in both pediatric- and adult-onset CVS. There were no significant differences in response to treatment in both groups of patients $(\mathrm{p}=0.27)$.

Pediatric-onset CVS patients differed from adult-onset CVS in having a higher prevalence of CVS-plus. (14\% vs. $3 \%, \mathrm{p}=0.05)$. There was a marked delay in diagnosis in both groups but those with pediatric-onset CVS has a much longer mean delay in diagnosis in comparison to adult- onset CVS $(10 \pm 7$ vs. $5 \pm 7$ years, $\mathrm{p}=0.001)$. Adult-onset CVS patients had a significantly higher incidence of tobacco and narcotic use of $46 \%$ and $23 \%$ when compared to $12 \%$ and $0 \%$ in the pediatric-onset group ( $\mathrm{p}=0.001$ and 0.004 respectively).

\section{Treatment profile of patients with CVS and variables} associated with response to treatment

Most patients 70/92 (76\%) were on tricyclic antidepressants (TCA) such as amitriptyline or nortriptyline, 18 /92 patients (20\%) on topiramate and 27/92 (30\%) on coenzyme Q-10, L-carnitine or riboflavin. Of the 77 patients with adequate data on response to triptans as abortive therapy, $64(83 \%)$ patients were able to abort episodes. Of the 70 patients on tricyclic antidepressants, 18 patients (26\%) had to stop the medication due to drug intolerance; the most common adverse effects included bad dreams, behavioral changes and increased somnolence. The mean dose of TCA used in patients who tolerated therapy was $83.3 \pm 36.6 \mathrm{mg}$. Adequate follow up data was available in $76 / 101(75 \%)$ patients (75\%) who were initiated on medical therapy and mean duration of follow-up was $11.2 \pm 6.2$ months. Response to treatment in these patients is depicted in figure 1. The majority of patients responded to treatment with 44 (58\%) patients having a complete response, $21(28 \%)$ a partial response and $11(14 \%)$ patients with no response to therapy. There were no significant differences in the type of medications or dosages used in complete, partial and non-responders. (Figure 2)

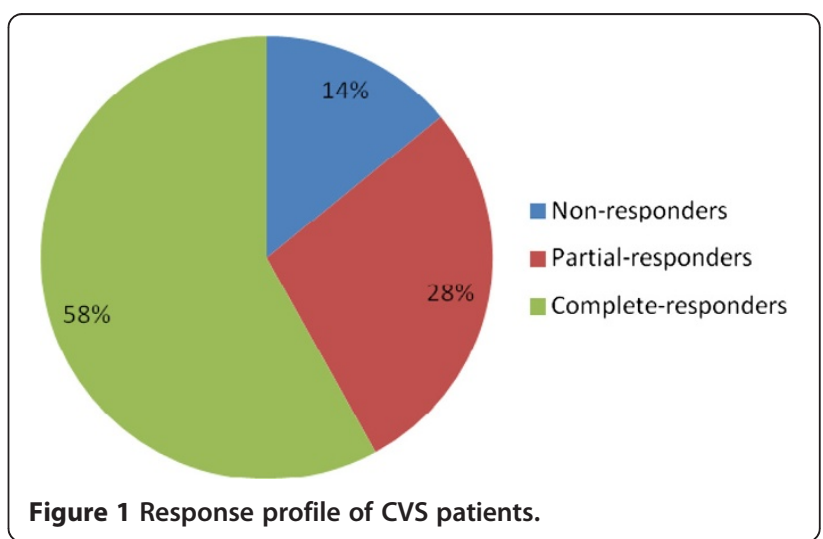




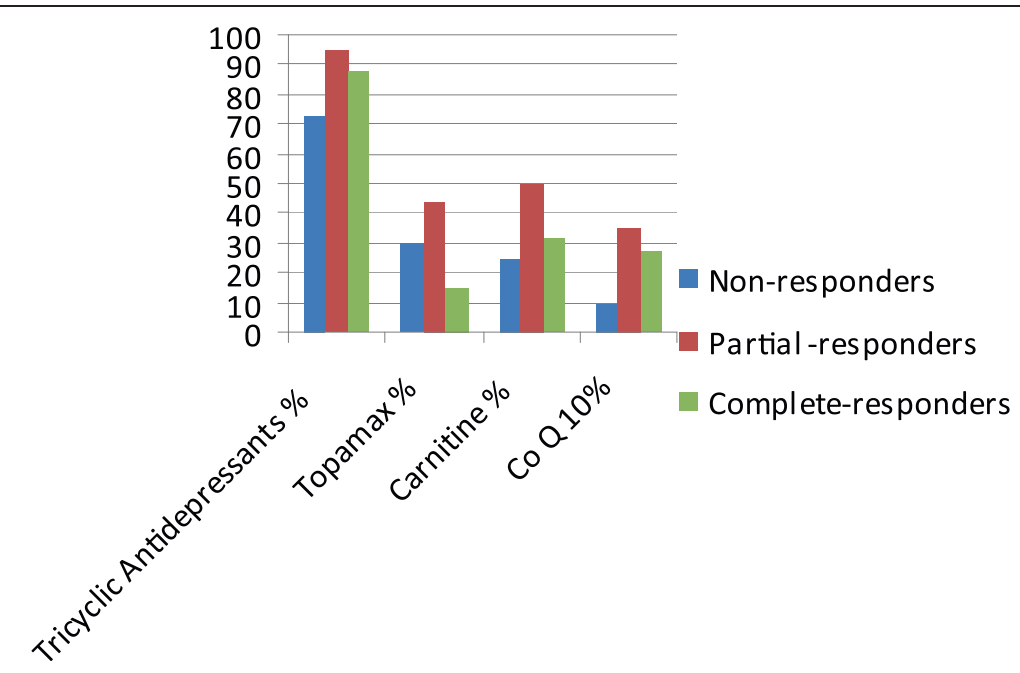

Figure 2 Medications used in complete, partial and non-responders did not reveal any significant differences.

The demographics and clinical characteristics of responders (both complete and partial) and non-responders are depicted in table 2. On univariate analysis, nonresponders were more likely to have a pattern of coalescence $(82 \%$ vs. $34 \% \mathrm{p}=0.005)$. They also had had longer

\section{Table 2 Univariate analysis of responders and} nonresponders to treatment

\begin{tabular}{|c|c|c|c|}
\hline Variables & $\begin{array}{l}\text { Responders } \\
\text { [Partial n-21; } \\
\text { Complete n-44] }\end{array}$ & $\begin{array}{l}\text { Non-Responders } \\
(n-11)\end{array}$ & $p$-value \\
\hline Pediatric onset & 31 & 18 & 0.49 \\
\hline Gender & 63 & 64 & 1 \\
\hline \multicolumn{4}{|l|}{ Females \% } \\
\hline \multicolumn{4}{|l|}{ Race } \\
\hline White \% & 82 & 82 & 1 \\
\hline Black \% & 14 & 18 & 0.66 \\
\hline Coalescence \% & 34 & 82 & 0.005 \\
\hline Narcotics \% & 13 & 45 & 0.02 \\
\hline Compliance \% & 88 & 38 & 0.004 \\
\hline Loss of job \% & 31 & 57 & 0.21 \\
\hline Disability \% & 20 & 57 & 0.05 \\
\hline Surgery for CVS \% & 29 & 30 & 1 \\
\hline No. of episodes/yr & $31.3 \pm 50$ & $46.6 \pm 52.8$ & 0.4 \\
\hline Duration of episodes (days) & $3.5 \pm 3.5$ & $6.5 \pm 3.5$ & 0.008 \\
\hline Warning phase (Minutes) & $159.2 \pm 365$ & $47.1 \pm 378.1$ & 0.45 \\
\hline Recovery Phase (days) & $1.7 \pm 1.9$ & $2.2 \pm 1.9$ & 0.43 \\
\hline No. of emesis/hr & $12.2 \pm 17.1$ & $19.1 \pm 17.4$ & 0.31 \\
\hline ED visits last year & $3.4 \pm 9$ & $15.8 \pm 9.2$ & 0.0002 \\
\hline Number of hospitalizations & $11.5 \pm 31.8$ & $31 \pm 32$ & 0.08 \\
\hline $\begin{array}{l}\text { Average duration } \\
\text { of hospitalizations }\end{array}$ & $5.1 \pm 8.8$ & $3.3 \pm 8.8$ & 0.57 \\
\hline
\end{tabular}

episodes lasting 6.5 days vs. 3.5 days and greater numbers of ED visits ( 15 vs. 3 ) in the year prior to evaluation compared to responders. The incidence of chronic opiate use was significantly higher amongst non-responders than responders $(46 \%$ vs. $13 \%, p=0.02)$. A significantly higher rate of disability amongst non-responders was also noted ( $57 \%$ vs. $20 \%, p=0.05$ ). Non-responders were less likely to be compliant to treatment ( $38 \%$ vs. $88 \%, \mathrm{p}=0.004$ ). On multivariate analysis only compliance was found to be statistically significant in predicting response to treatment ( $\mathrm{p}=0.03$, OR 9.6, 95 \% CI 1.18-77.05).

\section{Discussion}

The salient findings of our study are that both adultand pediatric-onset CVS patients have a similar demographic profile and disease characteristics except that pediatric-onset patients were more likely to be female and had a higher prevalence of CVS-plus with concomitant neurological disorders. This finding may be explained by the genetic differences that have been elucidated in previous studies $[2,10,11]$. The pediatric-onset group also had a lower incidence of tobacco and opiate use and a significantly longer delay in diagnosis of CVS. This longer delay in diagnosis is an unexpected finding given that CVS is fairly well-recognized condition in children. It should be noted that though our center attracts both children and adults with CVS, the majority of the patients with pediatric-onset CVS were not transitioned from the Children's Hospital of Wisconsin, which is also a tertiary center for pediatric CVS. Dysautonomia was diagnosed in $64 \%$ of our CVS patients which we recently reported to be a significant problem in this population [17]. Similar to reported literature, CVS patients in both groups had a strong personal or family history of migraine [18,19]. Co-morbid conditions including 
anxiety, depression and irritable bowel syndrome were seen in both groups and it is unclear if this is related to the lack of receiving a credible diagnosis and the inordinate delay in treatment that these patients experience. In addition, $30 \%$ of patients underwent invasive surgery for CVS symptoms without any benefit. Long delays in diagnosis along with such unnecessary and unhelpful interventions are unacceptable both from a patient standpoint and from an economic perspective.

The majority of our patients (86 \%) had either a complete or partial response to prophylactic medications with TCA's, topiramate and mitochondrial therapy such as carnitine, coenzyme Q-10 and ribofavin. The dose of carnitine used was 1 gram twice daily, co-enzyme Q-10 was $200 \mathrm{mg}$ twice daily and riboflavin $100 \mathrm{mg}$ once daily. On univariate analysis, non-response to therapy was associated with coalescence of symptoms, chronic opiate use and more severe disease as characterized by longer episodes, greater number of ED visits in the year prior to presentation, presence of disability and noncompliance. Compliance was the only significant variable on multivariate analysis that predicted response to therapy. Patients were deemed noncompliant if they continued to use marijuana and it is the practice of the author to advise complete abstinence from marijuana in all patients. We did not routinely check for cessation of marijuana in our patients with toxicology screens. There was no difference in response to therapy between pediatric-onset and adult-onset CVS patients despite recent reports of genetic differences. This may imply that there are other factors involved in the pathogenesis of this disorder aside from mitochondrial abnormalities in adults.

The few patients who were administered TCA's prior to being seen at our clinic were on a low daily dose of $25 \mathrm{mg}$ that is employed in other functional gastrointestinal disorders. Dose escalation to $1 \mathrm{mg} / \mathrm{kg}$ as used in children produced the desired therapeutic effect. However, there was a high incidence of side effects associated with TCA therapy resulting in discontinuation in $26 \%$ of patients which limited its use [20].

Marijuana use was seen in more than a third of patients with CVS and patients frequently reported using this for alleviation of nausea and as an appetite stimulant. There did not appear to be any clear causeand-effect or temporal relationship between the use of marijuana and onset of symptoms based on clinical history and these patients were not thought to have symptoms caused by the use of marijuana. Marijuana use has been thought to result in cannabinoid hyperemesis [2123]. Though the patients reported in the literature have not had adequate follow up; this entity as a separate disorder is subject to controversy. This raises questions about whether chronic marijuana use down regulates
CB1 receptors and paradoxically causes more nausea and vomiting which will need to be evaluated in the future studies.

We acknowledge that there are several limitations to this study as this was a retrospective analysis though the data was also obtained prospectively with a standardized questionnaire used in clinic. The response to medications has been arbitrary in CVS thus far and there has been no consensus or accepted definitions in adult patients for measures of response to treatment in CVS. ED utilization has been recently studied in CVS patients; we used overall improvement of symptom frequency and severity to assess response to treatment [24]. We were unable to report on exact numbers of ED visits prior to and after treatment as many patients visited other ED's for CVS episodes. In addition while other authors have measured ED visits and hospitalizations as outcomes of therapy, some patients with CVS have home intravenous fluid therapy or are seen in infusion clinics as in our practice to help control their symptoms and avoid long waits in the ED and prevent hospitalizations. Our study is one of the largest studies characterizing adult-onset and pediatric-onset CVS patients. This study should pave the way for future prospective evaluation of treatment in patients with CVS and standardized measures of disease outcomes.

\section{Conclusion}

In conclusion, adult-onset CVS patients have similar symptoms to those with pediatric- onset CVS and have similar rates of response to therapy. CVS patients appear to respond favorably to higher doses of TCA's of $1 \mathrm{mg} / \mathrm{kg}$ in comparison to the lower doses that are used in other functional gastrointestinal disorders. CVS is associated with an enormous psychosocial and economic burden and patients must be diagnosed early and treated with appropriate medications. Combination of medical and behavioral therapy based on a bio-psycho-social model is key to successfully treating patients.

\section{Methods}

\section{Study population}

A retrospective review of 101 adults with CVS seen at the Medical College of Wisconsin between September 2006 and October 2008 was performed. The adult CVS clinic at the Medical College of Wisconsin (MCW) serves as a tertiary referral center for patients with CVS. Patients were identified based on the Rome III criteria for CVS and comprised of patients from 15 states in the USA and Canada. Data was collected at the time of initial patient visit and subsequent follow up visits. All patients had stereotypical episodes at onset but some patients developed coalescence of symptoms later when they developed significant interepisodic nausea and 
dyspepsia and lost the typical periodicity seen during the initial phase of their illness. Patients with coalescence of symptoms were included only if they had episodic symptoms at the outset.

\section{Data collection}

Demographic characteristics including age, gender, race, geographic location, personal and family history of all patients were collected at the time of initial evaluation with a standard questionnaire that was completed by patients prior to their appointment. This was then reviewed at the time of the initial clinic visit and information was confirmed. Data was collected on disease characteristics such as duration and severity of disease, age of onset, delay in diagnosis, specific triggers, associated co-morbid conditions, history of marijuana use and number of emergency department (ED) visits and hospitalizations. Medications used as prophylactic therapy in CVS including tricyclic antidepressants such as amitriptyline and nortriptyline, anti-epileptics such as topiramate and mitochondrial supplements such as coenzyme Q-10, L-carnitine, and riboflavin were recorded. Adverse effects of medications, response to treatment, disability and job loss due to CVS were ascertained. Data was collected on a Microsoft Excel spreadsheet; most of the variables were in a categorical format with some data such as number of ED visits and hospitalizations recorded as continuous variables. Data was collected as per the historical recollection of the patient with supplementation from previous medical records. The patients were divided based on time of onset of symptoms (pediatric-onset and adult-onset) and response to therapy [1]. This study was approved by Institutional Review Board at the Medical College of Wisconsin.

All patients seen in our clinic were asked standard questions about the frequency, duration and severity of episodes. Complete response to therapy was defined as $\geq 80 \%$ amelioration in symptom duration, frequency and severity and partial response was at least a 50-80 \% reduction. Non-responders had either no change in their disease status or $<50 \%$ reduction in symptom duration, frequency and severity. Patients were deemed compliant if they reported taking their prescribed medications and either discontinued or reduced their marijuana intake significantly. Cessation of marijuana use was not verified routinely with a drug screen.

\section{Data analysis}

The data was analyzed using the Stata (StataCorp, College Station, TX). Categorical variables were summarized using proportions while continuous variables were summarized using means and standard deviation. The Fisher's exact test or the chi-square test was used to perform between-group comparisons of categorical variables. The $\mathrm{t}$-test was used for comparing continuous variables.

Univariate logistic regression was performed to identify variables associated with the dichotomous outcomes of interest, namely pediatric onset disease and response (complete / partial response vs. non-response) to therapy. Linear regression was used for continuous outcomes including number of hospitalizations, ED visits, duration of episodes and number of episodes. Variables that were significant in these regression models at $\mathrm{p}<0.1$ were selected for inclusion in the final multivariate model where $\mathrm{p}<0.05$ was indicative of independent statistical significance.

\section{Abbreviations}

CVS: Cyclic Vomiting Syndrome; ED: Emergency Department; MCW: Medical College of Wisconsin; TCA: Tricyclic Antidepressant; OR: Odds Ratio:

$\mathrm{Cl}$ : Confidence Interval.

\section{Competing interests}

The authors declare that they have no competing interests.

\section{Acknowledgement}

We would like to acknowledge the Cyclic Vomiting Syndrome Association for contributing towards publications costs.

\section{Author details}

${ }^{1}$ Department of Gastroenterology and Hepatology, Medical College of Wisconsin, 4th Floor, Froedtert East Building, 9200 W Wisconsin Avenue, Milwaukee, WI 53226, USA. ${ }^{2}$ Mayo Clinic Florida, Florida, USA. ${ }^{3}$ Wayne State University, Detroit, USA. ${ }^{4}$ Massachusetts General Hospital, Massachusetts, USA.

\section{Author contribution}

NK Acquisition of the data Analysis and interpretation of the data Drafting of the manuscript Revisions and corrections. QB Collection of data Organization of the data Review of charts and follow-up. NR Acquisition of the data Review of literature Drafting of the tables/figures. JS Administrative, technical, or material support Organization of the data Review of literature. AA Statistical Analysis Critical review of manuscript. AS Acquisition of data Patient recruitment and follow-up. WJH Study concept and design Critical review of the manuscript. TV Study concept and design Drafting of manuscript Critical review of the manuscript Study overview.

Received: 17 December 2011 Accepted: 28 May 2012

Published: 28 May 2012

\section{References}

1. Abell TL, Adams KA, et al: Cyclic vomiting syndrome in adults. Neurogastroenterol Motil 2008, 20(4):269-284.

2. Boles RG, Zaki EA, et al: Are pediatric and adult-onset cyclic vomiting syndrome (CVS) biologically different conditions? Relationship of adultonset CVS with the migraine and pediatric CVS-associated common mtDNA polymorphisms 16519T and 3010A. Neurogastroenterol Motil 2009, 21(9):936-e72

3. Fleisher DR, Matar M: The cyclic vomiting syndrome: a report of 71 cases and literature review. J Pediatr Gastroenterol Nutr 1993, 17(4):361-369.

4. Tack J, Talley NJ, et al: Functional gastroduodenal disorders. Gastroenterology 2006, 130(5):1466-1479.

5. Pareek N, Fleisher DR, et al: Cyclic vomiting syndrome: what a gastroenterologist needs to know. Am J Gastroenterol 2007, 102(12): 2832-2840.

6. Prakash C, Clouse RE: Cyclic vomiting syndrome in adults: clinical features and response to tricyclic antidepressants. Am J Gastroenterol 1999, 94 (10):2855-2860.

7. Fleisher DR, Gornowicz B, et al: Cyclic Vomiting Syndrome in 41 adults: the illness, the patients, and problems of management. BMC Med 2005, $3: 20$. 
8. Prakash C, Staiano A, et al: Similarities in cyclic vomiting syndrome across age groups. Am J Gastroenterol 2001, 96(3):684-688.

9. Boles RG, Lovett-Barr MR, et al: Treatment of cyclic vomiting syndrome with co-enzyme Q10 and amitriptyline, a retrospective study. BMC Neurol 2010, 28:10-10.

10. Camilleri M, Carlson P, et al: Mitochondrial DNA and gastrointestinal motor and sensory functions in health and functional gastrointestinal disorders. Am J Physiol Gastrointest Liver Physiol 2009, 296(3):G510-G516.

11. Zaki EA, Freilinger T, et al: Two common mitochondrial DNA polymorphisms are highly associated with migraine headache and cyclic vomiting syndrome. Cephalalgia 2009, 29(7):719-728.

12. Li BU, Lefevre F, et al: North American Society for Pediatric Gastroenterology, Hepatology, and Nutrition consensus statement on the diagnosis and management of cyclic vomiting syndrome. J Pediatr Gastroenterol Nutr 2008, 47(3):379-393.

13. Boles RG, Adams $\mathrm{K}$, et al: Maternal inheritance in cyclic vomiting syndrome. Am J Med Genet A 2005, 133A(1):71-77.

14. Venkatesan T, Wagler S, et al: M2013 Cyclic Vomiting Syndrome: Quantitative Pedigree Analysis for Maternal Inheritance Patterns in Adults and Children. Gastroenterology 2010, 138(5):S-458. Supplement 1.

15. Andersen JM, Sugerman KS, et al: Effective prophylactic therapy for cyclic vomiting syndrome in children using amitriptyline or cyproheptadine. Pediatrics 1997, 100(6):977-981.

16. Boles RG, Powers AL, et al: Cyclic vomiting syndrome plus. J Child Neurol 2006, 21(3):182-188.

17. Venkatesan T, Prieto $\mathrm{T}$, et al: Autonomic nerve function in adults with cyclic vomiting syndrome: a prospective study. Neurogastroenterol Motil 2010, 22(12):1303-1307.

18. Fleisher DR: Cyclic vomiting syndrome and migraine. J Pediatr 1999, 134 (5):533-535.

19. Li BU, Murray RD, et al: Is cyclic vomiting syndrome related to migraine? J Pediatr 1999, 134(5):567-572.

20. Hejazi RA, Reddymasu SC, et al: Efficacy of tricyclic antidepressant therapy in adults with cyclic vomiting syndrome: a two-year follow-up study. J Clin Gastroenterol 2010, 44(1):18-21.

21. Allen $\mathrm{JH}$, de Moore GM, et al: Cannabinoid hyperemesis: cyclical hyperemesis in association with chronic cannabis abuse. Gut 2004, 53 (11):1566-1570.

22. Ochoa-Mangado $E$, Jimenez Gimenez $M$, et al: Cyclical hyperemesis secondary to cannabis abuse. Gastroenterol Hepatol 2009, 32(6):406-409.

23. Sannarangappa V, Tan C: Cannabinoid hyperemesis. Intern Med J 2009, 39 (11):777-778.

24. Venkatesan T, Tarbell $S$, et al: A survey of emergency department use in patients with cyclic vomiting syndrome. BMC Emerg Med 2010, 10:4.

doi:10.1186/1471-230X-12-52

Cite this article as: Kumar et al:: Cyclic Vomiting Syndrome (CVS): is there a difference based on onset of symptoms - pediatric versus adult?. BMC Gastroenterology 2012 12:52.

\section{Submit your next manuscript to BioMed Central and take full advantage of:}

- Convenient online submission

- Thorough peer review

- No space constraints or color figure charges

- Immediate publication on acceptance

- Inclusion in PubMed, CAS, Scopus and Google Scholar

- Research which is freely available for redistribution 